\title{
Comparative Survey of Signal Processing and Artificial Intelligence Based Channel Equalization Techniques and Technologies
}

\author{
John Martin Ladrido, Jonnel Alejandrino, Emmanuel Trinidad, and Lawrence Materum \\ Department of Electronics and Communications Engineering, De La Salle University, Philippines \\ john_martin_ladrido@dlsu.edu.ph
}

\begin{abstract}
This paper presents an inspection and review of wired and wireless channel equalization techniques and their existing hardware implementations in terms of features, similarities, and differences. The authors begin with the theory behind channel equalization followed by techniques, and the technological realizations for achieving the proper filter in response to variations of the channel. Included in both the techniques and realizations are the rebirth of the use of artificial intelligence as a self-learning filter for the weights to use by the filtering structure of channel equalizers. These equalizers were compared, contrasted, and their key differentiation was identified. It was found that gaps such as complexity and convergence time are potential areas for extending the performance and limits of existing channel equalizers.
\end{abstract}

Key words: Equalizers, Adaptive Equalizers, Blind Equalizers, Channel Estimation, Decision Feedback Equalizers, Filters.

\section{INTRODUCTION}

As technology advances, data traffic in wired and wireless connections continue to rise. It is projected that in 2022, the annual global IP traffic is projected to be 396 exabytes per month or 4.8 zettabytes per year [1]. The higher the information density in storage devices, the more digital symbols are pack in time and space resulting in intersymbol interference or ISI. In order to mitigate this ISI, it will require some equalization. Each type of communication channels and data storage devices has its own characteristics and specifications, which leads to finding for the right channel equalization a challenge or a research topic of interest [2].

This paper presents an introduction to the problems that are being encountered when data is transmitted in a digital channel and focuses on the different types of channel equalization algorithms, technologies, and hardware that are used to mitigate these problems. In digital communication systems, noise and intersymbol interference (ISI) are the main problems that increase the bit error rate (BER). As technology advances further, and in order to mitigate these problems, new equalization algorithms are undergoing extensive research. As new applications are being developed, traditional equalization techniques are not enough to accommodate the present large bandwidth technologies such as $5 \mathrm{G}$, data-center applications, and the cloud. To achieve a channel that could supplement the needs of these applications, we will have to maximize the potential bandwidth that our current medium such as wired, wireless, and optical channels could provide. By choosing the best equalization algorithm that has the lowest or optimal BER results, these channels will be utilized in its optimum capacity. Thus, these channels at the same time would be able to provide more bandwidth.

In order to understand more regarding the attributes that are causing these channel impairments and how to mitigate this, the channel, noise, and intersymbol interference is discussed in section 2 and followed by a thorough discussion of an adaptive filter's mechanism in section 2.1. In the meantime, section 1.1 lists the recent surveys and comparative studies on channel equalization and discusses the deficiencies of these previous surveys and comparative studies regarding channel equalization. Finally, section 1.1 gives a summary of the review and its implications.

\subsection{EXISTING COMPARATIVE SURVEYS AND STUDIES}

There is a multitude of studies that were conducted for specific applications and structures by running comparative tests in predetermined channels or parameters such as modulation scheme and frequency. A list of conducted studies are as follows: Jaya and Vinodha [3] conducted a study using particle swarm optimization for finding the optimum solution, Randhawa, Sharma, and Dubey [4] conducted a study in designing an effective equalizer for multiple-input, multiple-output-orthogonal frequency-division multiplexing (MIMO-OFDM) systems, Elkassimi, Safi, and Manaut [5] created two algorithm to addressed blind channel equalization problem, Janjanam, Nunna, and Naraharisetti [6] performed a comparative analysis between particle swarm optimization (PSO) and teaching-learning based optimization (TLBO), Peng et al. compared three types of turbo equalization (maximum a posterior (MAP), frequency domain equalization (FDE), minimum mean-squared error (MMSE)) in detecting faster-than-Nyquist (FTN) signals [7], Moussa et al. proposed two blind equalization methods based on optimal bounding ellipsoid algorithms [8], Oyerinde [9] performed a comparative performance of equalizers for linear constellation pre-coded coherent optical orthogonal 
frequency-division multiplexing systems, Sharma, and Mishra [10] performed a comparative analysis of different adaptive equalizer algorithms in varying frequency scenarios, while Soni and Agarwal [11] performed a comparative analysis of different adaptive equalizer algorithms under different modulation techniques. All of the studies mentioned above performed comparative studies and surveys with varying conditions and without the use of an artificial neural network-based equalization algorithm. However, there had been few that conducted a comparative study in channel equalization using artificial neural networks (ANN) as an equalization algorithm.

Mota, Leal, and de Castro Lima [12], performed an evaluation of different neural networks algorithms such as, multilayer perceptron (MLP), functional link artificial neural network (FLANN), polynomial perceptron network (PPN), recurrent neural network (RNN), and genetic algorithm (GA) using different types of training algorithm such as backpropagation algorithm. An individual neural network algorithm was tested to verify their capability to specific problems. The performance showed a varying result as the learning algorithm needed to match to each structure to achieved optimum results. Regarding the neural weights, a genetic algorithm was able to perform better compared to other neural networks.

Chen et al. [13], performed a comparative analysis between complex-value (CV) B-spline neural network and complex-value polynomial-based neural network applied to nonlinear iterative frequency-domain decision feedback equalization (NIFDDFE) of Hammerstein communication channel. Results show that the two have comparable computational complexity. However, complex-value B-spline nonlinear iterative frequency-domain decision feedback equalization outperforms the complex-value polynomial-based nonlinear iterative frequency-domain decision feedback equalization in terms of accurately identifying and inverting Hammerstein systems

Zahid and Meng [14] conducted a comprehensive survey in neural network techniques such as multilayer perceptron, fuzzy neural network, functional link neural network, Chebyshev neural network, radial basis function neural network, and recursive neural networks with the addition of comparative computational complexity of its corresponding training algorithms. Results show the recursive neural network equalizers are superior compared to other variants, such as functional link artificial neural network being high in complexity, multilayer perceptron taking a lot of time for training, and radial basis function neural network limited usability in blind equalization. In the end, they recommended using support vector machines (SVM) for non-linear recursive channel equalization, and further research for the best training algorithm.

Zhou et al. [15] made a comparative study between different types of equalization technologies specific for the application of short-reach optical links using 4-level pulse-amplitude modulation (PAM4) with three variants of laser transmitters, electro-absorption modulator integrated with distributed feedback laser (EML), vertical-cavity surface-emitting laser (VCSEL), and directly modulated laser (DML). Based on the results, linear impairments such as bandwidth limitation and chromatic dispersion could be addressed by the feed-forward equalizer (FFE), and decision feedback equalizer (DFE). However, for nonlinear distortions, it would take a Volterra nonlinear equalizer (VNLE) and sparse VNLE to mitigate these channel impairments. For severe nonlinear distortions, support vector machines and neural networks-based equalizer are used. In the end, they had pointed out the potential of machine learning becoming the mainstream technology for future short-reach optical 4-level pulse-amplitude modulation transmission.

These previous studies that were conducted have their limitations for its specific application, like a comparative study of several equalization algorithms was conducted for the purpose of optical media using specific modulation or another study was conducted for different types of neural networks for Blind equalization. All of the studies were conducted using simulation such as MATLAB software. These open for the need to list comprehensive equalization algorithms regardless of its channel attributes, such as but not limited to the modulation scheme, media, frequency, and applications. As studies were conducted using simulation, these also open the need to list the currently available hardware that could be used to perform channel equalization.

This paper aims to provide a survey of the following: filter technology, equalization hardware, and equalization algorithms. The following Tables will be used for comparative analysis. Based on current filter technology, implemented or in production equalization schemes are always linear. There is no known equalization hardware as of this writing that is using neural networks-based equalization algorithm. There are several programmable decision feedback equalizers in the market, however, most of them are being configured using conventional equalization algorithms. The paper ends with an analysis of current existing equalization algorithms which points to neural networks or machine learning as the next-generation equalization technology.

The next chapter (chapter 2) will discuss the theory behind channel equalization. It will explain more about the channel, noise, intersymbol interference, and the adaptive filter. In chapter 3, channel equalization technologies will be enumerated and discussed. Here we will notice that the majority of existing equalization technologies are using a conventional equalization algorithm. Chapter 4 will be a brief discussion regarding the currently available equalization hardware in the market. This chapter list sample hardware coming from different vendors that are using either fixed or programmable algorithm. In chapter 5, a comprehensive list of equalization algorithms with their specific characteristics will be thoroughly discussed. In this chapter, we will be 
focusing more on artificial intelligence-based equalization as the purpose of this paper is to open up further studies for next-generation equalization algorithms. In chapter 6 , a brief summary and comparative analysis of the 5 Tables (filter technologies, equalization hardware, and equalization algorithms). And in chapter 7 will be the conclusion of this paper on how will the artificial intelligence-based equalization be the next-generation equalization algorithm and why it is superior to other traditional or conventional equalization algorithms.

\section{REVIEW OF CHANNEL EQUALIZATION THEORY}

In digital communications, the 4 main components are a transmitter, receiver, channel or medium, and the desired signal or data. When a signal passes through a channel, noise is added or introduced to the received signal. However, if the SNR or signal to noise ratio is high, a matched filter would be enough to mitigate this noise. For Gaussian noise, Shannon-Hartley Capacity Theorem in Eq. (1) shows that capacity $C$ of the channel is equal to the bandwidth (B) multiplied by log base 2 of 1 plus signal to noise ratio (SNR). The Shannon-Hartley Capacity Theorem shows that the information rate is limited by channel capacity. Base on this, it is important to note that there is a limitation in increasing the symbol rate beyond the Nyquist rate and addressing the resulting intersymbol interference by equalization [2].

$$
C=B \log _{2}(1+\mathrm{SNR})
$$

Intersymbol interference is interference between symbols that occurs at the receiver. In multiple channels or when multiple digital symbols are sent to the receiver, intersymbol interference or ISI is introduced. Sometimes, to enable higher data rates, partial response pulse shaping is used at the transmitter which introduced intersymbol interference as well. In summary, intersymbol interference is developed by transmitting more symbols than independent dimensions [2].

To better understand what is intersymbol interference, it is equated in Eq. (2) and Eq. (3) on how transmitted and received signal is. Let us consider the following: transmitted signal $\mathrm{s}(\mathrm{t}), \mathrm{u}(\mathrm{t})$ as the basic waveform for transmitting a sequence of symbols, $\mathrm{T}$ for the symbol interval, and to send $b_{n}$ (n as nth symbol), we send $b_{n} u(t-n T)$ as shown in Eq (2) [2], [16].

$$
s(t)=\sum_{n} b_{n} u(t-n T)
$$

The received signal $r(t)$ is shown in Eq. (3) based on a dispersive channel model. In a symbol, $\mathrm{u} * \mathrm{~h}_{\mathrm{c}}(\mathrm{t})$ is shown as the received waveform or received waveform for a symbol $\mathrm{v}(\mathrm{t}), \mathrm{u}(\mathrm{t})$ stands for transmitted signal and $\mathrm{h}_{\mathrm{c}}(\mathrm{t})$ is the impulse response of the channel. The desired signal or symbol $b_{o}$ is transmitted, and as it is received at the receiver, unwanted contributions are added coming from the symbol $b_{n}$ or other symbols or what is called intersymbol interference or ISI plus the noise $n(t)$. Equalization is introduced in order to mitigate this intersymbol interference. The main function of an equalizer is to recover the correct transmitted symbols. The presence of intersymbol interference in the system introduces a high bit error rate or BER as it introduces errors and difficulties in determining the exact transmitted symbols. Thus, equalizers are also coined as filters, as equalizer's function is just to filter out the channel effects [2], [16].

$$
r(t)=\sum_{k=-\infty}^{\infty} b_{n} v(t-n T)+n(t)
$$

\subsection{ADAPTIVE EQUALIZER}

Present-day has been mutated to data, knowledge, and information acquiring systems, which includes audio, video, and data that must be certain in terms of reliability, authenticity, and speed. The different emerging challenges to achieve these demands in a digital communication system has a severe approach. The mobility of communicators, highly dependable systems unaffected by the disturbance and inconvenience caused due to multipath fading wireless channels is one of the cutting edges of today's system [16].

In achieving the goals of high data rate to supply the extremity of the enormous bandwidth technology materializing nowadays, multiple thoroughfares of inputs and outputs aggregation are being implemented. Through these schemes, linearly increasing the channel capacity leads to channel fading that can root into the presence of (intersymbol interference) ISI. It introduces failures, inaccuracy, and glitch conclusive to the output of the receiver. Up-to-date communication systems deal with these problems up to some intensity, and to discharge the signal paths and channel changes have become more complex [2].

Channel Equalization deals with the disruptive channel effects by filtering it out, hence equalization is also designated as "filtering or equalizer" as a filter. This must be "adaptive" on account of time-varying channels. The intersymbol interference establishes the main intrusion in achieving increased digital transmission rates with the required accuracy [2], [17].

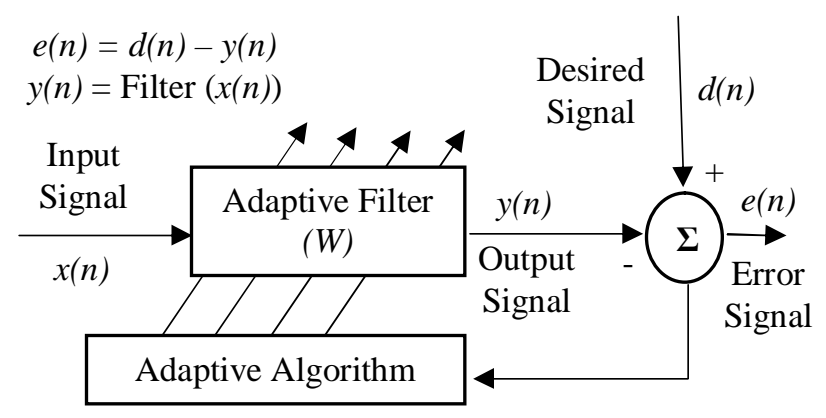

Figure 1: Simple block diagram of an adaptive equalizer 
John Martin Ladrido et al., International Journal of Emerging Trends in Engineering Research, 7(9), September 2019,311 - 322

Table 1: Equalization Techniques

\begin{tabular}{|c|c|c|}
\hline Type & Description & Algorithm/s \\
\hline Comb [18-20] & $\begin{array}{l}\text { implemented by combining a delayed duplicate of a signal with itself, resulting in a constructive } \\
\text { or destructive interference by using either Feedforward - Finite Impulse Response or Feedback - } \\
\text { Infinite Impulse Response }\end{array}$ & Feedforward, Feedback \\
\hline $\begin{array}{l}\text { Wiener Filter } \quad \text { or } \\
\text { Wiener-Kolmogorov } \\
{[12],}\end{array}$ & $\begin{array}{l}\text { uses the application of minimum mean square error (MMSE) criterion to minimize the error } \\
\text { between a desired and random process }\end{array}$ & $\begin{array}{l}\text { Minimum Mean-Square } \\
\text { Estimation (MMSE) }\end{array}$ \\
\hline $\begin{array}{l}\text { Matched or North [2], [21], } \\
{[22]}\end{array}$ & $\begin{array}{l}\text { correlates known delayed signal with an unknown signal in order to identify the template within } \\
\text { the unknown signal and maximize the output signal to noise ratio (SNR) }\end{array}$ & $\begin{array}{l}\text { Matrix Algebra, Lagrangian, } \\
\text { Least Squares Estimator }\end{array}$ \\
\hline $\begin{array}{l}\text { Kalman Filter or } \\
\text { Stratonovich-Kalman-Bucy } \\
{[17],[23]}\end{array}$ & $\begin{array}{l}\text { provides estimates and updating these estimates using weighted average by recursive-in-time } \\
\text { solution, resulting to higher accuracy by using two steps: step } 1 \text { is prediction, for which the } \\
\text { estimate is produced using a process equation at time instant } n-1 \text {; and step } 2 \text { is filtering, which } \\
\text { takes the produced estimate and updates it according to the process equation of an observed } \\
\text { signal at time instant } n\end{array}$ & Linear Quadratic Estimation \\
\hline Savitzky-Golay [24], [25] & $\begin{array}{l}\text { a digital filter used to increase the precision of the data and smoothing it, without distorting the } \\
\text { signal by applying it to a set of digital data points using regression analysis to minimize the sum } \\
\text { of squares corresponding to the linear functions of the data }\end{array}$ & Linear Least Squares \\
\hline $\begin{array}{l}\text { Kernel Adaptive }[17], \\
{[26-28]}\end{array}$ & $\begin{array}{l}\text { minimizes the errors of signal properties by minimizing the loss function through the adaptation } \\
\text { process by learning sequential sample signals using an online algorithm such as LMS and RLS }\end{array}$ & $\begin{array}{l}\text { Least Mean Squares (LMS), } \\
\text { Recursive Least Squares (RLS) }\end{array}$ \\
\hline $\begin{array}{l}\text { Hodrick-Prescott }[29], \\
{[30]}\end{array}$ & $\begin{array}{l}\text { applied in the raw data to minimize or remove the fluctuations and produced a smoothed-curve } \\
\text { representation of a time series by removing its cyclical component }\end{array}$ & $\begin{array}{l}\text { Hodrick-Prescott } \\
\text { Decomposition }\end{array}$ \\
\hline Turbo Equalizer [31-33] & $\begin{array}{l}\text { used to minimize the intersymbol interference (ISI) added to a signal that passes through } \\
\text { dispersive channels and the partial response of modems by implementing LMS, MAP, or BCJR }\end{array}$ & $\begin{array}{l}\text { Least Mean Square (LMS), } \\
\text { Maximum a Posteriori (MAP) } \\
\text { or Bahl, Cocke, Jelinek, and } \\
\text { Raviv (BCJR) }\end{array}$ \\
\hline $\begin{array}{l}\text { Kolmogorov-Zurbenko } \\
\text { [34], [35] }\end{array}$ & $\begin{array}{l}\text { a low-pass filter used to eliminate spectral leakage by being implemented as a special window } \\
\text { function which uses two parameters used in a series of iterations: moving average window and } \\
\text { number of iterations of the moving average itself }\end{array}$ & Kolmogorov-Zurbenko \\
\hline
\end{tabular}

The main purpose of an adaptive equalizer in Figure 1 is to adapt or self-learning as to which the input signal $x(n)$ is being processed by the adaptive filter, producing the output signal $y(n)$ which is then subtracted from the desired signal $d(n)$ in order to remove channel impairments by updating the weights or adaptive filter that will minimize the error signal $e(n)$. The adaptive filter is usually composed of multiple weights $(W)$ in order to achieve optimum results or minimum error signal as shown in Figure 2.

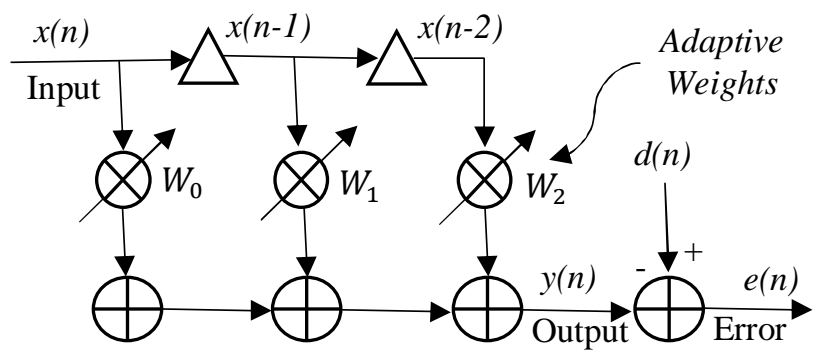

Figure 2: Block Diagram of an Adaptive Filter with multiple weights

\section{CHANNEL EQUALIZATION TECHNIQUES}

The advent purpose of this comparative study is to scope and compare distinct existing filtering methodologies, algorithms, and extant hardware under equalization to dispute against ISI. The study outlines the aspect of equalization in removing ISI and gives a comprehensive description and comparison of all the existing filters and equalizers in their respective environments.

The first stride of the paper is a review of all the existing main filtering technologies and their algorithm constraints before the application of advanced nonlinear algorithms. Filter technology is to dismiss out-of-band interference preceding to low-noise amplification in the receiver and intersymbol interference in the received signal. While in the transmitter, the filter is utilized to transmit a priori information for supervised equalization, and both receive and transmit filters are synchronized due to this set of data being transmitted by the filter at the transmitter. Resulting in the adjustment of the adaptive filter at the receiver. In the absence of this mechanism or supervised equalization leads to unsupervised or blind techniques [17].

Table 1, shows that the majority of the existing filters are adaptive equalizers implementing minimum mean-squared error, and least-squares algorithms. These algorithms have been implemented due to its low-complexity and low convergence time. Based on Table 1, we could already conclude that advanced algorithms such as artificial intelligence, machine learning, and support vector machines are not yet out in the market or being implemented in production.

Although filter technologies have an advantage of their capability to endure with time-invariant wireless channel attributes, refined processes are essential in channels to shorten and diminish the effects of intersymbol interference. During the process, channels must be adaptive because of the effects of the time variations and also for the channel to identify and regulate the changes. In the end, the ability and the achievable integrity of communication systems are intensely dependent on the system's learning technique or equalization algorithm concerning the channel's complexity. 
John Martin Ladrido et al., International Journal of Emerging Trends in Engineering Research, 7(9), September 2019,311 - 322

Table 2: Equalization Hardware

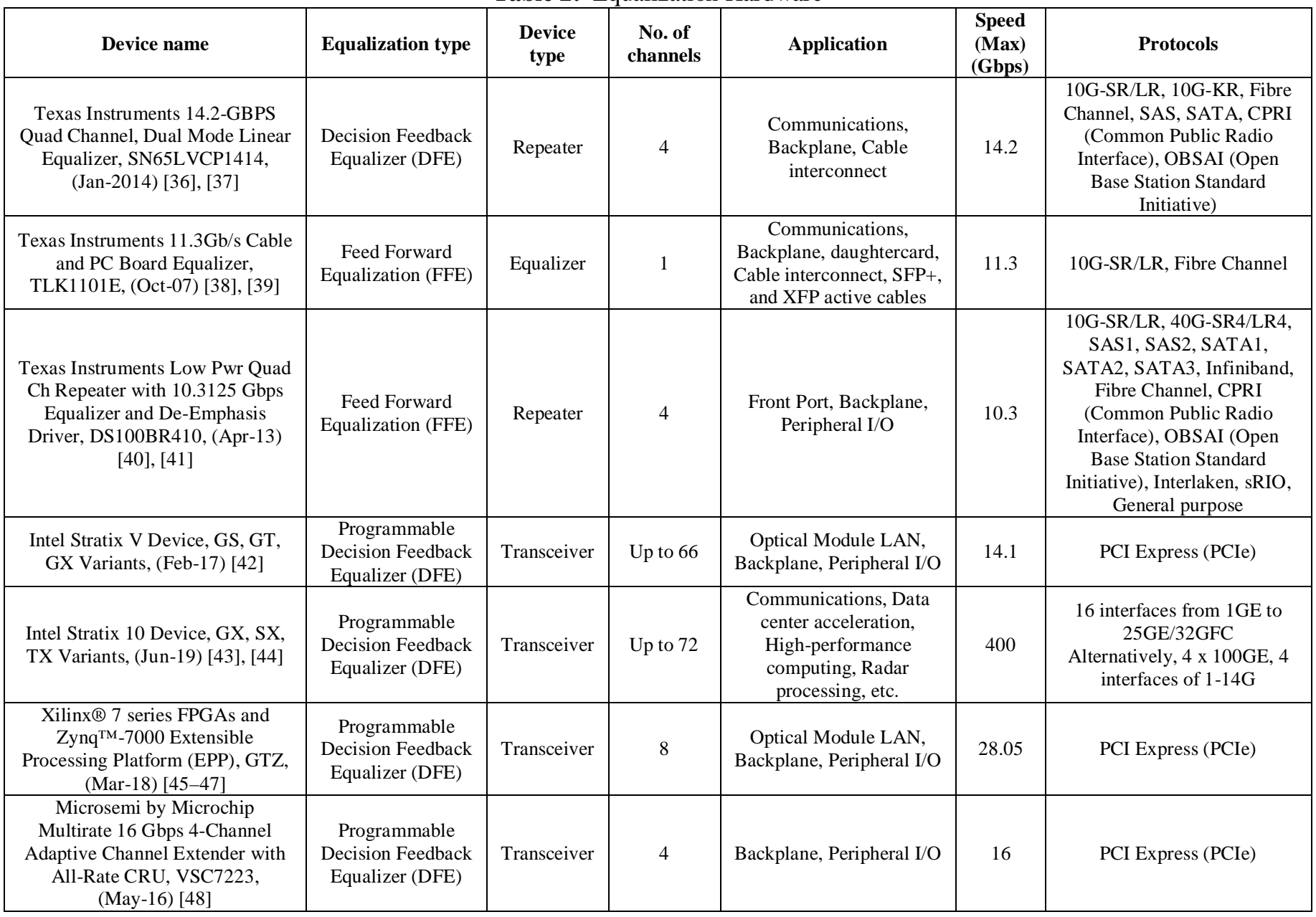

\section{HARDWARE REALIZATIONS OF CHANNEL} EQUALIZATION

Existing hardware and applications are also tabulated together with its specification and protocols as shown in Table 2. Accustomed hardware and equipment are narrowed to what is existing. Following the research of Murakawa et al. on the GRD chip (a group of 15 DSPs connected in a binary-tree network that implement a feed-forward neural network), the constraints because of complexity and cost in training sequence under the swarm algorithm results in an absence of material existence [49]. Jorge Pena et al. initiated self-reconfigurable adaptive systems that have the capability of adapting their own hardware configuration [50]. This administers development on performance, flexibility, and resources computational cost reductions. Its main downfall is its precondition of powerful optimization algorithms in order to search in a space of possible hardware configurations not sacrificing the complexity of the algorithm implemented on-chip. These scenarios only show that the condition of the interminable approach of a subsequential algorithm of equalization, as the same hardware equipment can have an extensive method, techniques, and algorithm. In Table 2, a sample of existing hardware models is listed with its corresponding vendor, equalization type, device type, sample application, bandwidth, and protocols. By definition, equalization type means what type of equalizer the device is, no. of channels corresponds to number of input and output ports it could support, application is the recommended usage or where it could be used by its corresponding vendor, speed is the maximum supported bandwidth of the channel of the corresponding device, and protocols are the supported mode of communication as per the device design or architecture. Table 2 shows that the older hardware implements a fixed equalization algorithm, whether it is decision feedback or feed-forward. However, the latest hardware or new variants shows that vendors are providing programmable decision feedback equalizer. This gives the users an option or open strategy of implementing or choosing the best equalization algorithm that would fit their systems and applications. This also gives the users the capability to test and evaluate latest equalization algorithms such as artificial intelligence, machine learning, or support vector machines if it would fit or improve their system functionality.

\section{ARTIFICIAL INTELLIGENCE BASED EQUALIZATION}

The inceptive or basic algorithm is introduced in the first part; it includes Linear and Non-linear Equalizer. These methods' general approach is to generally eliminate intersymbol interference. These are designed based on training methods to restrict and abrogate the outcome of the channel response. 
John Martin Ladrido et al., International Journal of Emerging Trends in Engineering Research, 7(9), September 2019,311 - 322

Table 3: Unsupervised/Blind Equalization Algorithms

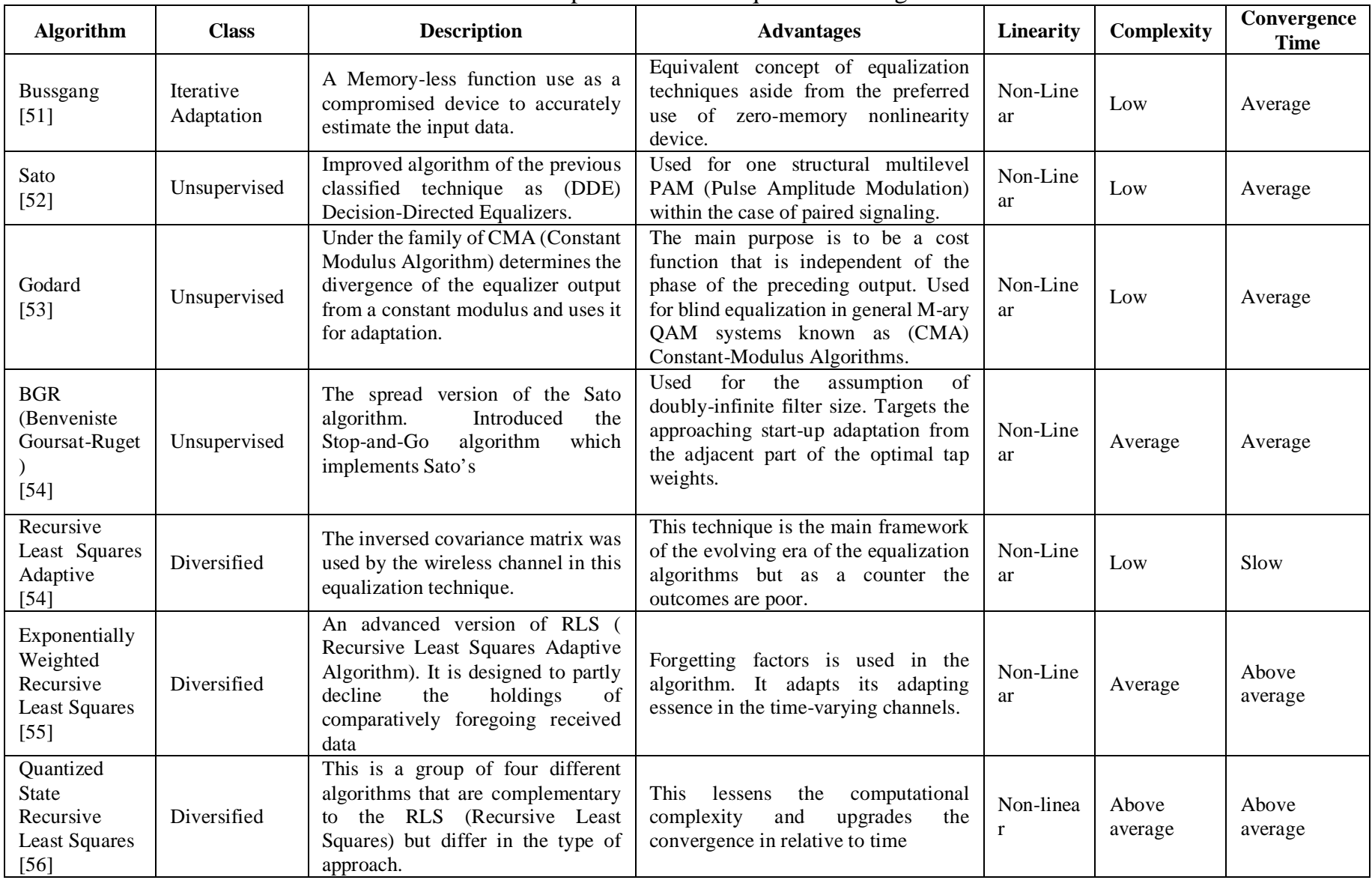

\section{ARTIFICIAL INTELLIGENCE BASED EQUALIZATION}

The inceptive or basic algorithm is introduced in the first part; it includes Linear and Non-linear Equalizer. These methods' general approach is to generally eliminate intersymbol interference. These are designed based on training methods to restrict and abrogate the outcome of the channel response. Training techniques aids to determine the convenient coefficients of the adaptive filter.There are two types of adaptive equalizer, the linear and non-linear. In linear, the system performs inferior to assertive channel conditions [57]. The zero forcing equalizer (ZF) and minimum mean square error equalizer (MMSE) are the linear equalizers.

While non-linear equalizer has a stage to change the successive outputs of the equalizer. The maximum likelihood sequence equalizer (MLSE), successive interference cancellation (SIC), and decision feedback equalizer (DFE) are nonlinear equalizers [58].

In different circumstances, blind equalization where no training period is required but is more complicated. This means that receiver decisions are used to generate an error signal. It is efficient in tracking slow variations in the channel but, it is not efficient during initial acquisition [59]. With the use of received signal sequence and input sequence statistics approach rather than training methods, it retrieves the unknown sequence bits to the remote channel [60], [61].
The complication with blind adaptive techniques is their poor convergence property. Customarily, a gradient descent-based algorithm is used with the blind adaptation schemes. The most commonly used gradient descent based blind adaptation algorithm is the constant modulus algorithm (CMA). Another popular adaptive blind equalization is the Bussgang algorithm (the Godard algorithm) or constant modulus (CMA) and fraction-spaced CMA algorithms [62].

The demand for high data-rates in wireless networks depends upon the resourceful capability of utilizing limited bandwidth available not sacrificing the high grade of mobility in diverse propagation environments. The orthogonal frequency division multiplexing (OFDM) methods have been engaged to employ this system predicament and also serves as a succeeding generation of the filter and channel equalization technologies. The main assistance of this method is minimizing the frequency nonselective or flat-fading, due to its narrow frequency signal will not be congested into a single part [70].

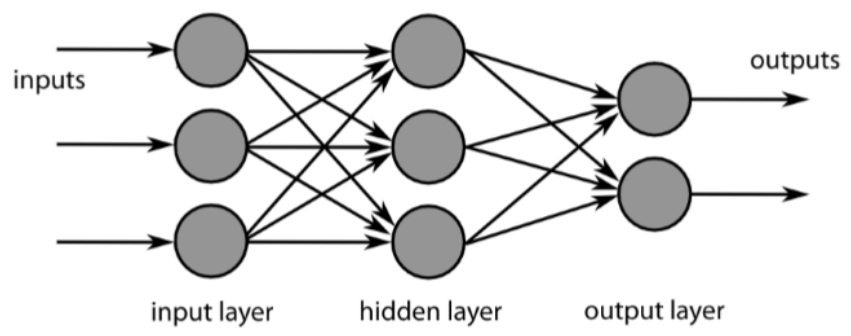

Figure 3: The architecture of the Neural Network 
Table 4: Adaptive Algorithm for Multiple System of Equalization

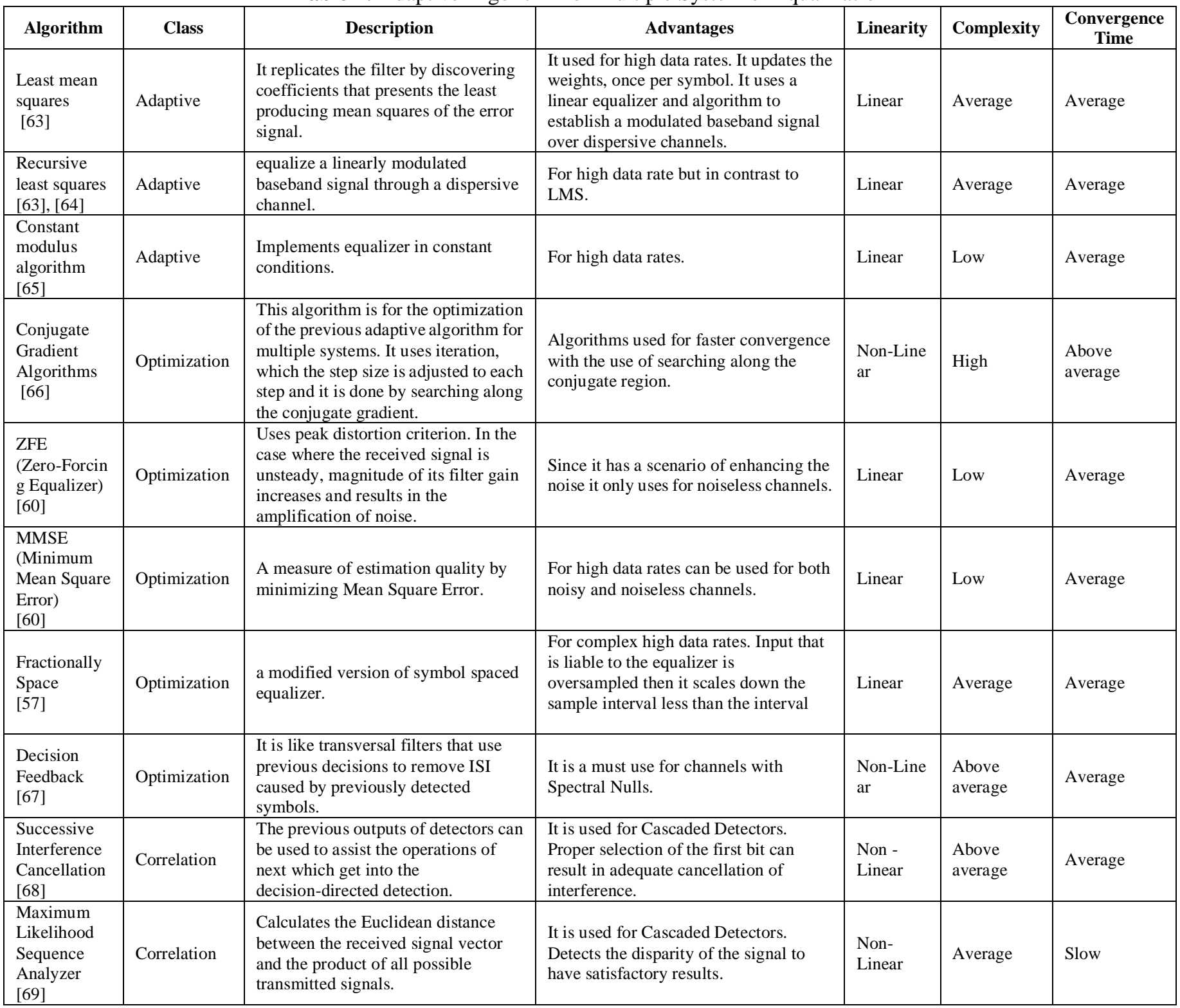

irreparable using a simple receiver. Thus, the complexity of the receiver structure and the multiplex computation of equalization algorithms are needed to estimate the channel. In this kind of equation, the need for equalizer algorithms arises. Some various equalization techniques that are used for OFDM systems are least mean squares (LMS), recursive least-squares algorithms (RLS), and constant modulus algorithm (CMA) [60].

Neural networks as shown in Figure 3 are a profound interconnection of a basic computational factor known as a perceptron, that are fundamental models of neurons in the human brain. Its architecture and calculation are utterly parallel networks of distinct computational elements systematized in correlate to each other. The main use of neural networks in equalization is the immense architecture of their parallel distributed components that support the network's computation complexity. The learning process in this kind of algorithm is apparent, in manner. It can produce accurate and reliable expected results or outputs [59].

Table 5 is divided into subsidiary groups that depend on their different corresponding types, starting from the initial type of unsupervised equalization algorithms up to recent experimentations on how to improve it in terms of complexity and convergence time. Description, advantages, linearity, complexity and convergence time is indicated in table 5. Complexity depends on the size of the signal alphabet, the memory of the ISI channel, the estimator window length, and the number of symbols transmitted over the channel. It is categorized to low, average, above average and high. Convergence time is the speed of the system to coincide with a relative signal with high integrity. It is categorized from slow, average, above average and fast. 
John Martin Ladrido et al., International Journal of Emerging Trends in Engineering Research, 7(9), September 2019,311 - 322

Table 5: Neural Network Techniques For Channel Equalization

\begin{tabular}{|c|c|c|c|c|c|c|}
\hline Algorithm & Class & Description & Advantages & Linearity & Complexity & $\begin{array}{c}\text { Convergence } \\
\text { Time }\end{array}$ \\
\hline $\begin{array}{l}\text { Back Propagation } \\
\text { [71] }\end{array}$ & Adaptive & $\begin{array}{l}\text { Basic training that is used to attain advanced } \\
\text { techniques of adaptive supervised training of } \\
\text { multilayer neural networks }\end{array}$ & For ease of hardware realization & Non-Linear & Low & Slow \\
\hline $\begin{array}{l}\text { Resilient } \\
\text { Backpropagation } \\
\text { [72] }\end{array}$ & Adaptive & $\begin{array}{l}\text { Are known as "squashing" they squeeze an } \\
\text { absolute input range into a definite output range } \\
\text { that makes it faster than the other. }\end{array}$ & $\begin{array}{l}\text { Deal with the problem of variation } \\
\text { in weights, bias and values when } \\
\text { training descent with sigmoid } \\
\text { functions. }\end{array}$ & Non-Linear & Average & Average \\
\hline $\begin{array}{l}\text { Fletcher-Reeves } \\
\text { [73] }\end{array}$ & Adaptive & $\begin{array}{l}\text { This is the ratio of the norm squared of the } \\
\text { current gradient to the norm squared of the } \\
\text { previous gradient. This way more fast than } \\
\text { variable learning rate backpropagation }\end{array}$ & $\begin{array}{l}\text { Accelerated technique of variable } \\
\text { learning rate backpropagation. } \\
\text { These algorithms are suitable for } \\
\text { networks having a large number of } \\
\text { weights }\end{array}$ & Non-Linear & $\begin{array}{l}\text { Above } \\
\text { average }\end{array}$ & $\begin{array}{l}\text { Above } \\
\text { average }\end{array}$ \\
\hline $\begin{array}{l}\text { Quasi-Newton } \\
\text { Algorithms } \\
\text { [74] }\end{array}$ & $\begin{array}{l}\text { Adaptive } \\
\text { optimization }\end{array}$ & $\begin{array}{l}\text { an alternative method of the conjugate gradient } \\
\text { methods in order to achieve fast optimization }\end{array}$ & $\begin{array}{l}\text { faster than conjugate gradient } \\
\text { methods but it is expensive and } \\
\text { complex to compute }\end{array}$ & Non-Linear & High & $\begin{array}{l}\text { Above } \\
\text { average }\end{array}$ \\
\hline $\begin{array}{l}\text { Shannon algorithm } \\
\text { [73] }\end{array}$ & Optimization & $\begin{array}{l}\text { approximate Algorithm need to be stored, whose } \\
\text { dimension is } n \times n \text {, where } n \text { is the total number of } \\
\text { weights and biases of the network }\end{array}$ & $\begin{array}{l}\text { more computation and storage are } \\
\text { required in each iteration, although } \\
\text { it normally converges in fewer } \\
\text { iterations }\end{array}$ & Non-Linear & High & $\begin{array}{l}\text { Above } \\
\text { average }\end{array}$ \\
\hline $\begin{array}{l}\text { Conjugate Gradient } \\
\text { Algorithms [75] }\end{array}$ & Optimization & $\begin{array}{l}\text { the step size is adjusted during each step } \\
\text { (iteration). They determine the step size by } \\
\text { searching along the conjugate gradient direction } \\
\text { which leads to degradation }\end{array}$ & $\begin{array}{l}\text { perform search along the conjugate } \\
\text { direction, which results in faster } \\
\text { convergence }\end{array}$ & Non-Linear & High & $\begin{array}{l}\text { Above } \\
\text { average }\end{array}$ \\
\hline $\begin{array}{l}\text { One Step Secant } \\
\text { Algorithm [76] }\end{array}$ & Optimization & $\begin{array}{l}\text { To fulfill the requirements of fewer } \\
\text { computations and smaller storage a secant } \\
\text { approximation is required }\end{array}$ & $\begin{array}{l}\text { has smaller storage and } \\
\text { computation requirements per each } \\
\text { iteration, but it requires a little more } \\
\text { computation and storage }\end{array}$ & Non-Linear & $\begin{array}{l}\text { Above } \\
\text { average }\end{array}$ & $\begin{array}{l}\text { Above } \\
\text { average }\end{array}$ \\
\hline $\begin{array}{l}\text { Levenberg-Marquardt } \\
\text { [73] }\end{array}$ & Optimization & $\begin{array}{l}\text { similar to the quasi-Newton methods, the } \\
\text { Levenberg-Marquardt algorithm was also } \\
\text { planned to approach the pace of second-order }\end{array}$ & $\begin{array}{l}\text { the fastest method for training } \\
\text { moderate-sized feedforward neural } \\
\text { networks }\end{array}$ & Non-Linear & Average & $\begin{array}{l}\text { Above } \\
\text { average }\end{array}$ \\
\hline $\begin{array}{l}\text { Multilayer perceptron } \\
\text { (MLP) [77] }\end{array}$ & Adaptive & $\begin{array}{l}\text { superior performance and symbol error rate but } \\
\text { suffer from a local minimum problem }\end{array}$ & $\begin{array}{l}\text { Attainment of nonlinear mapping } \\
\text { from input to output signals }\end{array}$ & Non-Linear & $\begin{array}{l}\text { Above } \\
\text { average }\end{array}$ & Average \\
\hline $\begin{array}{l}\text { Multilayer perceptron } \\
\text {-based Decision } \\
\text { Feedback [77] }\end{array}$ & Adaptive & $\begin{array}{l}\text { Used lattice filter with the application of } \\
\text { Decision Feedback Algorithm }\end{array}$ & $\begin{array}{l}\text { overcome the local minimum } \\
\text { problem }\end{array}$ & Non-Linear & $\begin{array}{l}\text { Above } \\
\text { average }\end{array}$ & Average \\
\hline $\begin{array}{l}\text { Complex Multilayer } \\
\text { perceptron } \\
\text { [77] }\end{array}$ & Adaptive & extended $\mathrm{BP}$ algorithm to the complex domain & $\begin{array}{l}\text { Equalization of complex QAM } \\
\text { signals }\end{array}$ & Non-Linear & High & Slow \\
\hline $\begin{array}{l}\text { Functional-link } \\
\text { Artificial Neural } \\
\text { Network } \\
{[78]} \\
\end{array}$ & Adaptive & $\begin{array}{l}\text { Introduces by the functional expansion of input } \\
\text { pattern by trigonometric polynomials }\end{array}$ & $\begin{array}{l}\text { For hardware with non-linear } \\
\text { Mapping rather than hidden layers }\end{array}$ & Non-Linear & High & Fast \\
\hline $\begin{array}{l}\text { Chebyshev Neural } \\
\text { Network } \\
\text { [79] }\end{array}$ & Adaptive & $\begin{array}{l}\text { Computationally efficient than using } \\
\text { trigonometric polynomials }\end{array}$ & $\begin{array}{l}\text { nonlinear dynamic system } \\
\text { identification. }\end{array}$ & Non-Linear & High & Fast \\
\hline $\begin{array}{l}\text { Reduced Decision } \\
\text { Feedback- } \\
\text { functional-link } \\
\text { Artificial Neural } \\
\text { Network [80] } \\
\end{array}$ & Adaptive & $\begin{array}{l}\text { the output signals are directly fed to the input } \\
\text { layer of the NN, instead of being taken as the } \\
\text { input signals of the network. }\end{array}$ & $\begin{array}{l}\text { To lower Hardware cost without } \\
\text { sacrificing system performance }\end{array}$ & Non-Linear & $\begin{array}{l}\text { Above } \\
\text { average }\end{array}$ & $\begin{array}{l}\text { Above } \\
\text { average }\end{array}$ \\
\hline $\begin{array}{l}\text { Stochastic-gradient } \\
\text { training algorithm } \\
\text { [81] }\end{array}$ & Adaptive & $\begin{array}{l}\text { weights and hidden-layer biases are randomly } \\
\text { and the output weights are then analytically } \\
\text { calculated. }\end{array}$ & $\begin{array}{l}\text { For improvement of RBF in blind } \\
\text { equalization }\end{array}$ & Non-Linear & $\begin{array}{l}\text { Above } \\
\text { average }\end{array}$ & $\begin{array}{l}\text { Above } \\
\text { average }\end{array}$ \\
\hline $\begin{array}{l}\text { Minimal resource } \\
\text { allocation } \\
\text { network (MRAN) } \\
{[82]} \\
\end{array}$ & Adaptive & $\begin{array}{l}\text { No hidden neurons. A pruning strategy that is } \\
\text { used to remove the hidden neurons that do not } \\
\text { contribute significantly to the output. }\end{array}$ & $\begin{array}{l}\text { neurons adjust the existing network } \\
\text { parameters according to the training } \\
\text { data received }\end{array}$ & Non-Linear & $\begin{array}{l}\text { Above } \\
\text { average }\end{array}$ & $\begin{array}{l}\text { Above } \\
\text { average }\end{array}$ \\
\hline $\begin{array}{l}\text { complex-valued } \\
\text { growing and pruning } \\
\text { (CGAP) RBF NN [83] }\end{array}$ & Adaptive & $\begin{array}{l}\text { the nearest neuron (based on the Euclidean } \\
\text { distance to the latest input data) is tested for its } \\
\text { significance, resulting in a more compact } \\
\text { network. }\end{array}$ & $\begin{array}{l}\text { for communication channel } \\
\text { equalization of four QAM }\end{array}$ & Non-Linear & High & $\begin{array}{l}\text { Above } \\
\text { average }\end{array}$ \\
\hline $\begin{array}{l}\text { Radial basis function } \\
\text { (RBF) [82] }\end{array}$ & Adaptive & $\begin{array}{l}\text { classifies the received signal according to the } \\
\text { class of the center closest to the received vector }\end{array}$ & $\begin{array}{l}\text { as a replacement for the sigmoidal } \\
\text { transfer function }\end{array}$ & Non-Linear & High & Fast \\
\hline $\begin{array}{l}\text { Recurrent Neural } \\
\text { Network-based } \\
{[84]}\end{array}$ & $\begin{array}{l}\text { Adaptive } \\
\text { variable }\end{array}$ & $\begin{array}{l}\text { IIR filters with feedback, outperform FNNs such } \\
\text { as MLP or RBF networks, ability to learn } \\
\text { nonlinear mappings of arbitrary complexity }\end{array}$ & $\begin{array}{l}\text { for fast-fading channels, solution } \\
\text { for non-linear blind equalization }\end{array}$ & Non-Linear & High & Fast \\
\hline $\begin{array}{l}\text { Dynamic Fuzzy Neural } \\
\text { Networks Dynamic [85] }\end{array}$ & $\begin{array}{l}\text { Adaptive } \\
\text { variable }\end{array}$ & $\begin{array}{l}\text { Combination of neural network learning } \\
\text { capabilities and fuzzy rules }\end{array}$ & $\begin{array}{l}\text { avail the advantages of both fuzzy } \\
\text { logic and neural networks }\end{array}$ & Non-Linear & High & Fast \\
\hline $\begin{array}{l}\text { Artificial Neural } \\
\text { Network based joint } \\
\text { temporal and spatial } \\
\text { equalization [86] }\end{array}$ & $\begin{array}{l}\text { Comparative } \\
\text { adaptation }\end{array}$ & $\begin{array}{l}\text { Designed to outperform the combination of the } \\
\text { temporal and spatial cross-talk }\end{array}$ & $\begin{array}{l}\text { For multiple input multiple output } \\
\text { visible light communication system }\end{array}$ & Non-Linear & High & Fast \\
\hline
\end{tabular}

Table 3 compares and surveys the different advances on Unsupervised or Blind Equalization Algorithms. These algorithms are responsible to have an effective approach in adaptively finding out the best possible weight vector of the equalizer, without prior knowledge to channel. Information and identity of the channel are required for decisive communication. This is not well-known and must be obtained commonly using some recursive adaptation algorithm. 
The essence of the advancement of technology and communications nowadays is to achieve the transfer of information immensely but rapidly. High data rates are in demand nowadays, in this generation of big data communication, an adaptive algorithm must also conform.

Table 4 shows the comparison of the different adaptive algorithms, in the continuance of the increase of demand in bandwidth and data rate. It can be adaptive in relation to dispersion of amplitude and phase. It can be an optimization of the previous algorithm. Lastly, it can be a correlation class that achieves elimination through interrelationship.

Due to the high demand for data rates, it results in the complexity and extended approach of parallel transmission level. Non-linear systems are the consequence, and neural networks can be adequate to decode transmitted symbols that are afflicted by fading channels. Classification can be done also by channel equalization. In the received symbol sequences spaces, the neural network can conveniently make decision sectors. Distinctively, neural networks have compelling efficiency of approximation and form decision sectors with forthwith shaped boundaries. These properties and descriptions support the neural network to concede and perform the task of a channel equalizer algorithm.

Table 5 shows the neural network section. This survey pursues to actuate the efficiency of several neural network training algorithms by comparing their attribute, process, and performance.

\section{RESULTS AND DISCUSSIONS}

Table 1 listed the available equalization technologies that are currently being implemented or in production. These current equalization technologies are implementing simple algorithms such as minimum mean-square error and least-squares. As of this writing, sophisticated algorithms such as artificial intelligence, machine learning, and support vector machines are still under evaluation or subjected to further research.

Table 2 shows that the latest equalization hardware has given the users to use whatever equalization algorithm that they see fit in their respective environments or applications, as the majority of the latest equalization hardware is now programmable.

In Tables 3, 4, and 5, the following results tabulated are established from different references and are systemized into groups of classifications. At the class column, each technique is classified as adaptive, unsupervised, diversified, optimization and correlation. This describes their categorization as a technique of algorithm used for equalization. Description and advantages show the considerable and dominant differences of each in differing aspects. Linearity shows where a function does the technique or algorithm are best used. Linear means that the equalizer is a linear function of its input and for non-linear, equalizer function is irrelevant to its source signal. Computational complexity is also considered and the analysis of it depends on the size of the signal variable used, the memory of the ISI channel, the estimator window length, and the number of symbols transmitted over the channel that is reviewed in the references of this paper. Last is the convergence time, and the survey references of this paper consider it as the length of training sequences that have been varied over a reasonable range to examine the results of each equalization.

\section{CONCLUSION}

Various technologies, methods, algorithms and the existing hardware for solving the problem of channel equalization is discussed, compared and outlined in distinction to its history and continuously arriving advancement. Filter technology is the ground of equalization techniques and currently being implemented. This uses primary algorithms such as minimum mean-square error and least-squares. Those techniques and technological realizations lead to hardware. In continuance of the increase of demand in bandwidth and data rate, different advanced algorithms develop.

The comparative survey determines that the equalization system's important attributes are on its computational complexity, class, length of the training sequence, and convergence speed of communication. A comparison of the recent studies shows that without an increment in the length of the training sequence results in a speed downturn. Hence, a transparent or less length of training symbols will result in a data rate increase. Computational complexity is also a constraint over the time of the communication. Those importunities can be reduced by computational estimation of synaptic weights that is possible to with artificial intelligence methods such as neural networks.

In reference to this study, the most effective technology, hardware available is the turbo equalizer, by the reason of its flexibility to advanced algorithms. For equalization algorithms, artificial neural networks based joint temporal and spatial equalization algorithm outperforms the other in terms of complexity. Whereas on, existing hardware, Intel Stratix 10 Device, GX, SX, TX Variants is recommendable because of its vast channels, faster convergence time and advanced protocol.

Future works may develop, and new hardware may materialize, maximizing the potential bandwidth that our medium can provide. Improvement on equalization must focus on the advances of algorithms and minimize the length of the training sequence by keeping the same performance of the system. 
John Martin Ladrido et al., International Journal of Emerging Trends in Engineering Research, 7(9), September 2019,311 - 322

\section{Acknowledgment}

De La Salle University is acknowledged for supporting this work.

\section{REFERENCES}

[1] V. Cisco, "Cisco visual networking index: Forecast and trends, 2017-2022," White Paper, vol. 1, 2018.

[2] G. E. Bottomley, Channel Equalization for Wireless Communications: From Concepts to Detailed Mathematics. Wiley, 2012. https://doi.org/10.1002/9781118105283

[3] S. Jaya and R. Vinodha, "Survey on Adaptive Channel Equalization Techniques using Particle Swarm Optimization," International Journal of Scientific Engineering and Technology, vol. 2, no. 9, pp. 849-852, 2013.

[4] N. S. Randhawa, S. Sharma, and R. K. Dubey, "A survey of equalization techniques for an effective equalizer design in MIMO-OFDM systems," in 2015 International Conference on Circuits, Power and Computing Technologies [ICCPCT-2015], 2015, pp. 1-5. https://doi.org/10.1109/ICCPCT.2015.7159297

[5] S. Elkassimi, S. Safi, B. Manaut, and S. Taj, "Comparative Study of Blind Channel Identification and Equalization Algorithms," in Proceedings of the New Challenges in Data Sciences: Acts of the Second Conference of the Moroccan Classification Society, 2019, p. 3.

[6] L. Janjanam, K. C. Nunna, and V. P. Naraharisetti, "A comparative analysis of channel equalization algorithms," in 2017 2nd IEEE International Conference on Recent Trends in Electronics, Information \& Communication Technology (RTEICT), 2017, pp. 349-353.

[7] S. Peng, A. Liu, X. Pan, X. Liang, and K. Wang, "Turbo equalization and detection for faster-than-nyqusit signaling: A comparative study," in 2016 8th International Conference on Wireless Communications \& Signal Processing (WCSP), 2016, pp. 1-6.

https://doi.org/10.1109/WCSP.2016.7752573

[8] A. Moussa, M. Pouliquen, M. Frikel, S. Bedoui, K. Abderrahim, and M. M'Saad, "Comparative study of blind equalizers based on optimal bounding ellipsoid algorithms under awgn and fading channels," in 2017 14th International Multi-Conference on Systems, Signals \& Devices (SSD), 2017, pp. 11-16.

[9] O. O. Oyerinde, "Comparative performance of equalizers for linear constellation precoded coherent optical OFDM systems," in 2017 11th International Conference on Signal Processing and Communication Systems (ICSPCS), 2017, pp. 1-6.

[10] N. Sharma and S. Mishra, "A comparative analysis of various adaptive equalizer algorithms for different frequency scenario," in 2017 International Conference on Innovations in Information, Embedded and Communication Systems (ICIIECS), 2017, pp. 1-4.
[11] S. Soni and A. Agarwal, "Analysis and synthesis of adaptive equalization techniques under various modulation techniques," in 2015 International Conference on Recent Developments in Control, Automation and Power Engineering (RDCAPE), 2015, pp. 102-106.

[12] T. A. Mota, J. F. Leal, and A. C. de Castro Lima, "Neural Equalizer Performance Evaluation Using Genetic Algorithm," IEEE Latin America Transactions, vol. 13, no. 10, pp. 3439-3446, 2015.

[13] S. Chen, X. Hong, E. F. Khalaf, F. E. Alsaadi, and C. J. Harris, "Comparative performance of complex-valued B-spline and polynomial models applied to iterative frequency-domain decision feedback equalization of Hammerstein channels," IEEE transactions on neural networks and learning systems, vol. 28, no. 12, pp. 2872-2884, 2016.

https://doi.org/10.1109/TNNLS.2016.2609001

[14] M. Zahid and Z. Meng, "Recent Advances in Neural Network Techniques for Channel Equalization: A Comprehensive Survey," in 2018 International Conference on Computing, Electronics Communications Engineering (iCCECE), 2018, pp. 178-182.

[15] H. Zhou, Y. Li, Y. Liu, L. Yue, C. Gao, W. Li, J. Qiu, H. Guo, X. Hong, Y. Zuo, and others, "Recent Advances in Equalization Technologies for Short-Reach Optical Links Based on PAM4 Modulation: A Review," Applied Sciences, vol. 9, no. 11, p. 2342, 2019.

[16] T.Wong and T.Lok, "Theory of Digital Communications 4. ISI \& Equalization Chapter 4 Intersymbol Interference and Equalization | Mohammad Firman - Academia.edu." 2004.

[17] J. M. T. Romano, R. Attux, C. C. Cavalcante, and R. Suyama, Unsupervised Signal Processing: Channel Equalization and Source Separation. CRC Press, 2018. https://doi.org/10.1201/b10370

[18] R. Russell, "Why be so overly concerned about comb filtering in column speakers when you probably get this all the time with the speakers y." 2006.

[19] J. O. Smith, "Feedforward Comb Filters." 2010.

[20] J. O. Smith, "Feedback Comb Filters." 2010.

[21] A. Fish, S. Gurevich, R. Hadani, A. Sayeed, and O. Schwartz, "Computing the matched filter in linear time," arXiv preprint arXiv:1112.4883, 2011.

[22] G. Turin, "An introduction to matched filters," IRE Transactions on Information Theory, vol. 6, no. 3, pp. 311-329, Jun. 1960.

[23] D. Ledenyov and V. Ledenyov, "On the Stratonovich-Kalman-Bucy Filtering Algorithm Application for Accurate Characterization of Financial Time Series with Use of State-Space Model by Central Banks," Available at SSRN 2594333, 2013.

[24] A. Savitzky and M. J. E. Golay, "Smoothing and Differentiation of Data by Simplified Least Squares Procedures.," Analytical Chemistry, vol. 36, no. 8, pp. 1627-1639, 1964.

[25] A. Savitzky, "A Historic Collaboration," Analytical Chemistry, vol. 61, no. 15, p. 921A-923A, 1989. 
[26] W. Liu, J. C. Principe, and S. Haykin, Kernel adaptive filtering: a comprehensive introduction, vol. 57. John Wiley \& Sons, 2011. https://doi.org/10.1002/9780470608593

[27] W. Liu, P. P. Pokharel, and J. C. Principe, "The Kernel Least-Mean-Square Algorithm," IEEE Transactions on Signal Processing, vol. 56, no. 2, pp. 543-554, Feb. 2008.

[28] Y. Engel, S. Mannor, and R. Meir, "The kernel recursive least-squares algorithm," IEEE Transactions on Signal Processing, vol. 52, no. 8, pp. 2275-2285, Aug. 2004.

[29] R. J. Hodrick and E. C. Prescott, "Postwar US business cycles: an empirical investigation," Journal of Money, credit, and Banking, pp. 1-16, 1997.

[30] M. O. Ravn and H. Uhlig, "On adjusting the Hodrick-Prescott filter for the frequency of observations," Review of economics and statistics, vol. 84, no. 2, pp. 371-376, 2002.

[31] M. Tuchler, R. Koetter, and A. C. Singer, "Turbo equalization: principles and new results," IEEE Transactions on Communications, vol. 50, no. 5, pp. 754-767, 2002.

[32] R. Koetter, A. C. Singer, and M. Tuchler, "Turbo equalization," IEEE signal processing magazine, vol. 21, no. 1, pp. 67-80, 2004.

[33] B. L. Yeap, T. H. Liew, J. Hamorsky, and L. Hanzo, "Comparative study of turbo equalization schemes using convolutional, convolutional turbo, and block-turbo codes," IEEE Transactions on Wireless Communications, vol. 1, no. 2, pp. 266-273, 2002.

[34] B. Close, I. Zurbenko, and M. B. Close, "Package 'kza," 2018.

[35] W. Yang and I. Zurbenko, "Kolmogorov-Zurbenko filters," Wiley Interdisciplinary Reviews: Computational Statistics, vol. 2, no. 3, pp. 340-351, 2010. https://doi.org/10.1002/wics.71

[36] Datasheet, "SN65LVCP1414," Texas Instruments, [2019-07-31], 2014.

[37] TexasInstruments, "SN65LVCP1414 14.2-GBPS Quad Channel, Dual Mode Linear Equalizer | TI.com.".

[38] TexasInstruments, "11.3-Gbps Cable and PC Board Equalizer (Rev. A)." 2007.

[39] Datasheet, "TLK1101E," 11.3-Gbps Cable and PC Board Equalizer, [2019-07-30], 2007.

[40] TexasInstruments, "DS100BR410 Quad Chan Repeatr w/10.3125 Gbps Equalzr \& De-Emphs Drvr datasheet (Rev. B).” 2013.

[41] Datasheet, "DS100BR410 Quad Chan Repeatr w/10.3125 Gbps Equalzr \& De-Emphs Drvr datasheet (Rev. B)," http://www.ti.com/lit/ds/symlink/ds100br410.pdf, 2013.

[42] Intel, "Stratix V Device Handbook: Volume 2: Transceivers." 2017.

[43] Intel, "Intel® Stratix ${ }^{\circledR} 10$ Device Design Guidelines." 2019.

[44] Intel, "Intel® Stratix® 10 FPGAs Applications." .

[45] P. Newson, "The application of fpgas for wireless base-s tation connectivity," 2014.
[46] H. Fu, "Equalization for high-speed serial interfaces in Xilinx 7 series FPGA transceivers," Xilinx white paper, vol. 419, 2012.

[47] M. Timmons, "High-Performance Machine Vision Systems Using Xilinx 7 Series Technology,” 2014.

[48] Microsemi, "VSC7223 | Microsemi.".

[49] M. Murakawa, S. Yoshizawa, I. Kajitani, X. Yao, N. Kajihara, M. Iwata, and T. Higuchi, "The grd chip: Genetic reconfiguration of dsps for neural network processing," IEEE Transactions on Computers, vol. 48, no. 6, pp. 628-639, 1999. https://doi.org/10.1109/12.773799

[50] J. Pena, A. Upegui, and E. Sanchez, "Particle swarm optimization with discrete recombination: an online optimizer for evolvable hardware," in First NASA/ESA Conference on Adaptive Hardware and Systems (AHS'06), 2006, pp. 163-170.

[51] A. Waseem and A. Hossain, "MIMO Channel Equalization and Symbol Detection using Multilayer Neural Network." 2013.

[52] Z.-Z. Ding, X.-D. Zhang, and Y.-T. Su, "Performance analysis of a nested RLS-type interference suppression algorithm for CDMA systems in dispersive channels," IEEE Signal Processing Letters, vol. 14, no. 1, pp. 1-4, 2006. https://doi.org/10.1109/LSP.2006.881531

[53] S. S. Haykin, Adaptive filter theory. Prentice Hall, 1991.

[54] P. M. Clarkson, Optimal and Adaptive Signal Processing. CRC Press, 2017.

[55] Y. Li and Z. Ding, Blind equalization and identification. CRC press, 2001.

[56] B. Widrow and E. Walach, Adaptive inverse control, reissue edition: a signal processing approach. John Wiley \& Sons, 2008.

[57] N. S. Randhawa and S. Khurana, "Error rate performance enhancement using hybrid equalization technique in MIMO-OFDM systems," in 2014 International Conference on High Performance Computing and Applications (ICHPCA), 2014, pp. 1-5.

[58] O. Simeone, Y. Bar-Ness, and U. Spagnolini, "Linear and nonlinear preequalization/equalization for MIMO systems with long-term channel state information at the transmitter," IEEE Transactions on Wireless Communications, vol. 3, no. 2, pp. 373-378, 2004. https://doi.org/10.1109/TWC.2003.821139

[59] R. Johnson, P. Schniter, T. J. Endres, J. D. Behm, D. R. Brown, and R. A. Casas, "Blind equalization using the constant modulus criterion: A review," Proceedings of the IEEE, vol. 86, no. 10, pp. 1927-1950, 1998.

[60] J. G. Proakis and M. Salehi, Digital communications, vol. 4. McGraw-hill New York, 2001.

[61] D. Godard, "Self-recovering equalization and carrier tracking in two-dimensional data communication systems," IEEE transactions on communications, vol. 28, no. 11, pp. 1867-1875, 1980.

[62] I. Fijalkow, C. Manlove, and C. Johnson, "Adaptive fractionally spaced blind CMA equalization: Excess MSE," IEEE Transactions on Signal Processing, vol. 46, no. 1, pp. 227-231, 1998. 
John Martin Ladrido et al., International Journal of Emerging Trends in Engineering Research, 7(9), September 2019,311 - 322

[63] A. Grace Dela Cruz, "Recursive Least Square and Least Means Square Equalizers Optimization Algorithms in Rayleigh Fading," International Journal of Advanced Trends in Computer Science and Engineering, vol. 8, pp. 689-695, 2019. https://doi.org/10.30534/ijatcse/2019/56832019

[64] A. Kulkarni and E. Rani, "Unconstraint 2-level cost optimization coding for signal processing," International Journal of Advanced Trends in Computer Science and Engineering, vol. 8, 2019. https://doi.org/10.30534/ijatcse/2019/15812019

[65] Y. Kao, S. Lee, C. Hsiao, and P. L. Chio, "One-tap frequency domain LMS equalizer for OFDM systems," in ISCOM, 2003.

[66] S. Mishra, R. Prusty, and P. K. Hota, "Analysis of Levenberg-Marquardt and Scaled Conjugate gradient training algorithms for artificial neural network based LS and MMSE estimated channel equalizers," in 2015 International Conference on Man and Machine Interfacing (MAMI), 2015, pp. 1-7.

[67] P. Li and R. C. De Lamare, "Adaptive decision-feedback detection with constellation constraints for MIMO systems," IEEE Transactions on Vehicular Technology, vol. 61, no. 2, pp. 853-859, 2011.

[68] H. Zhao, X. Zeng, J. Zhang, and T. Li, "Nonlinear adaptive equalizer using a pipelined decision feedback recurrent neural network in communication systems," IEEE Transactions on Communications, vol. 58, no. 8, pp. 2193-2198, 2010.

[69] N. S. Randhawa, "An Overview of Adaptive Channel Equalization Techniques and Algorithms," signal, vol. 8, p. 9, 2013.

[70] N. Sood, A. K. Sharma, and M. Uddin, "BER performance of OFDM-BPSK and-QPSK over generalized gamma fading channel," International Journal of Computer Applications, vol. 3, no. 6, pp. 13-16, 2010. https://doi.org/10.5120/736-1035

[71] M. Minsky and S. A. Papert, Perceptrons: An introduction to computational geometry. MIT press, 2017.

[72] A. Kantsila, M. Lehtokangas, and J. Saarinen, "Complex RPROP-algorithm for neural network equalization of GSM data bursts," Neurocomputing, vol. 61, pp. 339-360, 2004.

[73] M. T. Hagan, H. B. Demuth, M. H. Beale, and others, "Neural network design, PWS Pub," Co., Boston, vol. 3632, 1996.

[74] K. N. Paracha and A. Zerguine, "Quasi-Newton multimodulus blind equalization algorithm," in 2009 IEEE International Symposium on Signal Processing and Information Technology (ISSPIT), 2009, pp. $151-154$.

[75] S. Zhang, J. Liu, X. Zeng, J. Lu, Y. Wei, and M. Wang, "Analysis of a modified adaptive least mean square frequency-domain algorithm for equalization in polarization division multiplexed-mode division multiplexed fiber transmission," in 2017 Opto-Electronics and Communications Conference
(OECC) and Photonics Global Conference (PGC), 2017, pp. 1-3.

https://doi.org/10.1109/OECC.2017.8114862

[76] T. S. Rappaport and others, Wireless communications: principles and practice, vol. 2. prentice hall PTR New Jersey, 1996.

[77] S. Chen, G. Gibson, C. Cowan, and P. Grant, “Adaptive equalization of finite non-linear channels using multilayer perceptrons," Signal processing, vol. 20, no. 2, pp. 107-119, 1990.

[78] J. C. Patra, R. N. Pal, B. Chatterji, and G. Panda, "Identification of nonlinear dynamic systems using functional link artificial neural networks," IEEE Transactions on Systems, Man, and Cybernetics, part b (cybernetics), vol. 29, no. 2, pp. 254-262, 1999.

[79] J. C. Patra and A. C. Kot, "Nonlinear dynamic system identification using Chebyshev functional link artificial neural networks," IEEE Transactions on Systems, Man, and Cybernetics, Part B (Cybernetics), vol. 32, no. 4, pp. 505-511, 2002.

[80] W.-D. Weng, C.-S. Yang, and R.-C. Lin, "A channel equalizer using reduced decision feedback Chebyshev functional link artificial neural networks," Information Sciences, vol. 177, no. 13, pp. 2642-2654, 2007. https://doi.org/10.1016/j.ins.2007.01.006

[81] D. Hidalgo, O. Castillo, and P. Melin, "Type-1 and type-2 fuzzy inference systems as integration methods in modular neural networks for multimodal biometry and its optimization with genetic algorithms," Information Sciences, vol. 179, no. 13, pp. 2123-2145, 2009.

[82] D. Jianping, N. Sundararajan, and P. Saratchandran, "Communication channel equalization using complex-valued minimal radial basis function neural networks," IEEE Transactions on Neural Networks, vol. 13, no. 3, pp. 687-696, 2002.

[83] M.-B. Li, G.-B. Huang, P. Saratchandran, and N. Sundararajan, "Complex-valued growing and pruning RBF neural networks for communication channel equalisation," IEE Proceedings-Vision, Image and Signal Processing, vol. 153, no. 4, pp. 411-418, 2006.

[84] S. L. Goh and D. P. Mandic, "Recurrent neural networks with trainable amplitude of activation functions," Neural Networks, vol. 16, no. 8, pp. 1095-1100, 2003.

[85] M.-B. Li and M. J. Er, "Channel equalization using dynamic fuzzy neural networks," IFAC Proceedings Volumes, vol. 41, no. 2, pp. 4072-4077, 2008.

[86] S. Rajbhandari, H. Chun, G. Faulkner, H. Haas, E. Xie, J. J. McKendry, J. Herrnsdorf, E. Gu, M. D. Dawson, and D. O'Brien, "Neural Network-Based Joint Spatial and Temporal Equalization for MIMO-VLC System," IEEE Photonics Technology Letters, vol. 31, no. 11, pp. 821-824, 2019.

https://doi.org/10.1109/LPT.2019.2909139 